\title{
Reasoned and implicit processes in heavy episodic drinking: An integrated dual-process model
}

\author{
Kyra Hamilton ',2* (D), Isabelle Gibbs', Jacob J. Keech' (D) and \\ Martin S. Hagger ${ }^{1,3,4}$ iD \\ 'School of Applied Psychology, Menzies Health Institute Queensland, Griffith \\ University, Brisbane, Queensland, Australia \\ ${ }^{2}$ Laboratory of Self-Regulation and Health Psychology and Behavioural Medicine \\ Research Group, School of Psychology, Faculty of Health Sciences, Curtin University, \\ Perth, Western Australia, Australia \\ ${ }^{3}$ Psychological Sciences, University of California, Merced, California, USA \\ ${ }^{4}$ Faculty of Sport and Health Sciences, University of Jyväskylä, Jyväskylä, Finland
}

Objectives. University students commonly engage in heavy episodic drinking (HED), which contributes to injury risk, deleterious educational outcomes, and economic costs. Identification of the determinants of this risky behaviour may provide formative evidence on which to base effective interventions to curb HED in this population. Drawing from theories of social cognition and dual-process models, this study tested key hypotheses relating to reasoned and implicit pathways to action for HED in a sample of Australian university students who drink alcohol.

Design. A two-wave correlational design was adopted.

Methods. Students $(N=204)$ completed self-reported constructs from social cognition theories with respect to HED at an initial time point (TI): attitude, subjective norm, perceived behavioural control, intentions, habit, past behaviour, and implicit alcohol identity. Four weeks later (T2), students self-reported their HED behaviour and habit.

Results. An initial path model indicated attitude and subjective norm predicted intentions, and intentions and implicit alcohol identity predicted HED. Inclusion of past behaviour and habit revealed direct effects of these on HED. Effects of TI habit on HED were indirect through T2 habit, and there were indirect effects of past behaviour on HED through habit at both time points and the social cognition constructs. Direct effects of intentions and implicit alcohol identity, and indirect effects of attitude and subjective norm, on HED, were attenuated by the inclusion of past behaviour and habit.

Conclusion. Results indicate that university students' HED tends to be governed by non-conscious, automatic processes than conscious, intentional processes.

* Correspondence should be addressed to Kyra Hamilton, Health and Psychology Innovations (HaPl) Laboratory, School of Applied Psychology, Griffith University, Mt Gravatt Campus, 176 Messines Ridge Road, Mt Gravatt, Queensland, QId 4I22, Australia (email: kyra.hamilton@griffith.edu.au). 


\section{Statement of contribution}

What is already known on this subject?

- Social cognitive factors are associated with risky alcohol consumption behaviours.

- Dual-process models are being used to explain health behaviours, such as heavy episodic drinking (HED).

\section{What does this study add?}

- Past HED behaviour and HED habits have direct and indirect effects on students' HED behaviour.

- Past behaviour and habit attenuate the effects of intentions and implicit alcohol identity on HED.

Heavy episodic drinking (HED) is a high-risk pattern of drinking in which individuals consume a high amount of alcohol over a relatively short period of time. Globally, over 1 billion drinkers are heavy episodic drinkers (WHO, 2018). HED among young adults is particularly prevalent in high-income countries such as the United States, Canada, and Australia (WHO, 2018), and is especially common among university students. For example, in Australia, around 50\% of male and 35\% of female university students consumed alcohol at hazardous levels (Stafford, 2017). HED has been associated with deleterious effects including increased risk of injury and involvement in the incidence of interpersonal violence and social disorder, and poor academic outcomes (NHMRC, 2009; WHO, 2018). Definitions of HED vary by country and organization, but according to the WHO (2018) it is defined as consuming more than six 'standard' drinks (any alcoholic beverage containing $10 \mathrm{~g}$ of alcohol) in one session, at least monthly (WHO, 2018). The over-representation of university students engaging in HED and the consistently small effects of interventions aimed at curbing student HED (Prosser, Gee, \& Jones, 2018) emphasizes the need to identify the determinants and associated mechanisms driving this behaviour in this population. Recent research has applied social cognition theories to identify the psychological correlates of risky alcohol consumption behaviours, including HED, and the processes involved (Caudwell, Keech, Hamilton, Mullan, \& Hagger, 2019). A leading approach has been to adopt integrated models of social cognition that encompass constructs representing reasoned and implicit processes that lead to behavioural engagement (Strack \& Deutsch, 2004). Consistent with this approach, the present study focused on identifying the determinants of HED in a sample of university students based on this integrated approach. The research may assist in identifying the modifiable determinants that may be targeted in behavioural interventions to reduce HED incidence.

We propose a set of key hypotheses relating to constructs that represent reasoned and implicit pathways to action for HED in a sample of university students who were regular social drinkers. Specifically, our hypotheses were derived from an integrated social psychological model drawing from previous research on social cognition theories (Ajzen, 2011) and dual-process models (Strack \& Deutsch, 2004) applied to health behaviour. The proposed model is presented in Figure 1.

In the present model, reasoned processes are represented by the effects of the belief-based constructs and intentions from the theory of planned behaviour (Ajzen, 2011). Specifically, attitude, subjective norm, and perceived behavioural control are expected to predict HED mediated by intentions. However, consistent with previous research and theory, HED is characterized as an appetitive behaviour that is likely influenced by implicit processes that reflect non-conscious decision-making such as affect and situational cues, and affect behaviour beyond an individual's awareness 


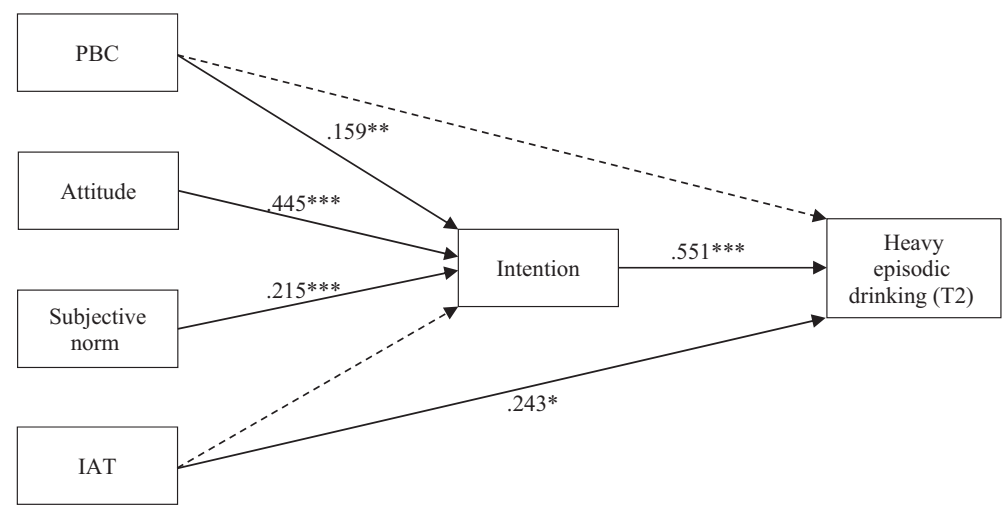

Figure I. Standardized parameter estimates and probability statistics for path analysis of integrated dualprocess model excluding habit and past behaviour. T2 = Measure taken at time point 2, 4 weeks after initial survey. ${ }^{* * *} p<.00 \mathrm{I} ;{ }^{* *} p<.0 \mathrm{I} ;{ }^{*} p<.05$.

(Caudwell et al., 2019). In the present model, we included two measures that reflect these implicit processes: self-reported habit and implicit alcohol identity. Implicit alcohol identity was proposed to reflect non-conscious evaluations of alcohol consumption developed through repeated experiences with alcohol. Self-reported habits reflect the extent to which individuals experience the behaviour as enacted beyond their awareness, efficiently and automatically, likely developed through frequent experience with the behaviour in the presence of stable contexts and cues (Orbell \& Verplanken, 2010). Consistent with the proposal that these constructs represent implicit approaches, we expect both constructs to have direct effects on HED behaviour bypassing intentions. Effects of these constructs may also attenuate the effects of the social cognition constructs to the extent that they reflect previous decision-making and rumination over performing the behaviour. In our model, we extended previous research examining habitual effects in social cognition theories (Allom, Mullan, Cowie, \& Hamilton, 2016; Hamilton, Kirkpatrick, Rebar, \& Hagger, 2017) by including measures of habit at both the initial time point and at follow-up alongside the measure of behaviour. Such effects may demonstrate the extent to which habits are stable for this behaviour, and account for past behaviour effects over time. Finally, we examined the effects of past behaviour in the model, conceptualized as previous frequency of participation in HED. Past behaviour has been proposed to model numerous non-conscious processes including habits and decisions based on implicit cognition or behavioural 'scripts' (Brown, Hagger, Morrissey, \& Hamilton, 2017; Ouellette \& Wood, 1998). We therefore expected past behaviour to have direct effects on subsequent behaviour. Furthermore, to the extent that habit and implicit alcohol identity mediated effects of past behaviour on subsequent behaviour, we will have evidence as to the types of non-conscious processes modelled by past behaviour. We therefore also expect indirect effects of past behaviour on subsequent HED over time through self-reported habit and implicit alcohol identity. Finally, we also expected the effects of habit and past behaviour to be mediated by intention and the social cognition constructs, consistent with the premise that these variables also reflect previous decisionmaking (Ajzen, 2002b). 


\section{Methods}

\section{Participants}

Participants $(N=204,68.63 \%$ female; mean age $=20.03$ years, $S D=2.15)$ were undergraduate university students. Participants were recruited using a combination of face-to-face and online methods. Face-to-face recruitment involved direct approach by a member of the research team, with the potential participant being given a flyer containing the study URL. Online recruitment included notices sent in broadcast emails to all students at the university, notices posted on Facebook, and a notice posted on the school subject/ participant pool. Participants were offered entry into a prize draw or course credit as an incentive for participation. Data were collected across two university semesters. Eligibility criteria included being aged between 18 and 25 years, a drinker of alcohol, an undergraduate student, and not currently pregnant.

\section{Design and procedure}

The study was conducted between May and November 2016. A two-wave prospective correlational design was used with participants visiting the research laboratory (55\%) or completing an online survey (45\%) at an initial time point (T1) and a single follow-up conducted remotely by email or telephone (T2). At T1, participants were asked to read a brief information passage and complete a consent form. Next, they were presented with a passage defining the target behaviour ('Heavy episodic "binge" drinking is consuming more than six standard drinks on a single occasion') and a pictorial guide providing examples a standard drink for common alcoholic beverages. Participants then completed self-report measures of social cognitive variables (intention, attitude, subjective norm, perceived behavioural control, habit, and past behaviour) presented using the Qualtrics ${ }^{\mathrm{TM}}$ online survey tool. Participants then completed a measure of implicit alcohol identity using an implicit association test (IAT) administered by the Inquisit ${ }^{\mathrm{TM}}$ experimental software. Participants were contacted 4 weeks to complete follow-up measures of selfreported frequency of HED and self-reported habit. Approval for study procedures was granted from the Griffith University Human Research Ethics Committee. All procedures were in accordance with the ethical standards of the institutional and/or national research committee and with the 1964 Helsinki declaration and its later amendments or comparable ethical standards. Informed consent was obtained from all individual participants included in the study.

\section{Measures}

Study measures were multi-item self-report measures of social cognitive constructs, habit, and past frequency of HED based on published guidelines and measures used in previous studies (Ajzen, 2002a; Caudwell et al., 2019; Gardner, Abraham, Lally, \& de Bruijn, 2012). Participants provided their responses on scales with between five- and seven-point response options (see Table 1).

\section{Social cognitive constructs}

Measures of constructs from the theory of planned behaviour were developed according to published guidelines (Ajzen, 2002a). Participants completed measures of intentions (e.g., 'I intend to engage in heavy episodic drinking in the next 4 weeks'), attitude (e.g., 


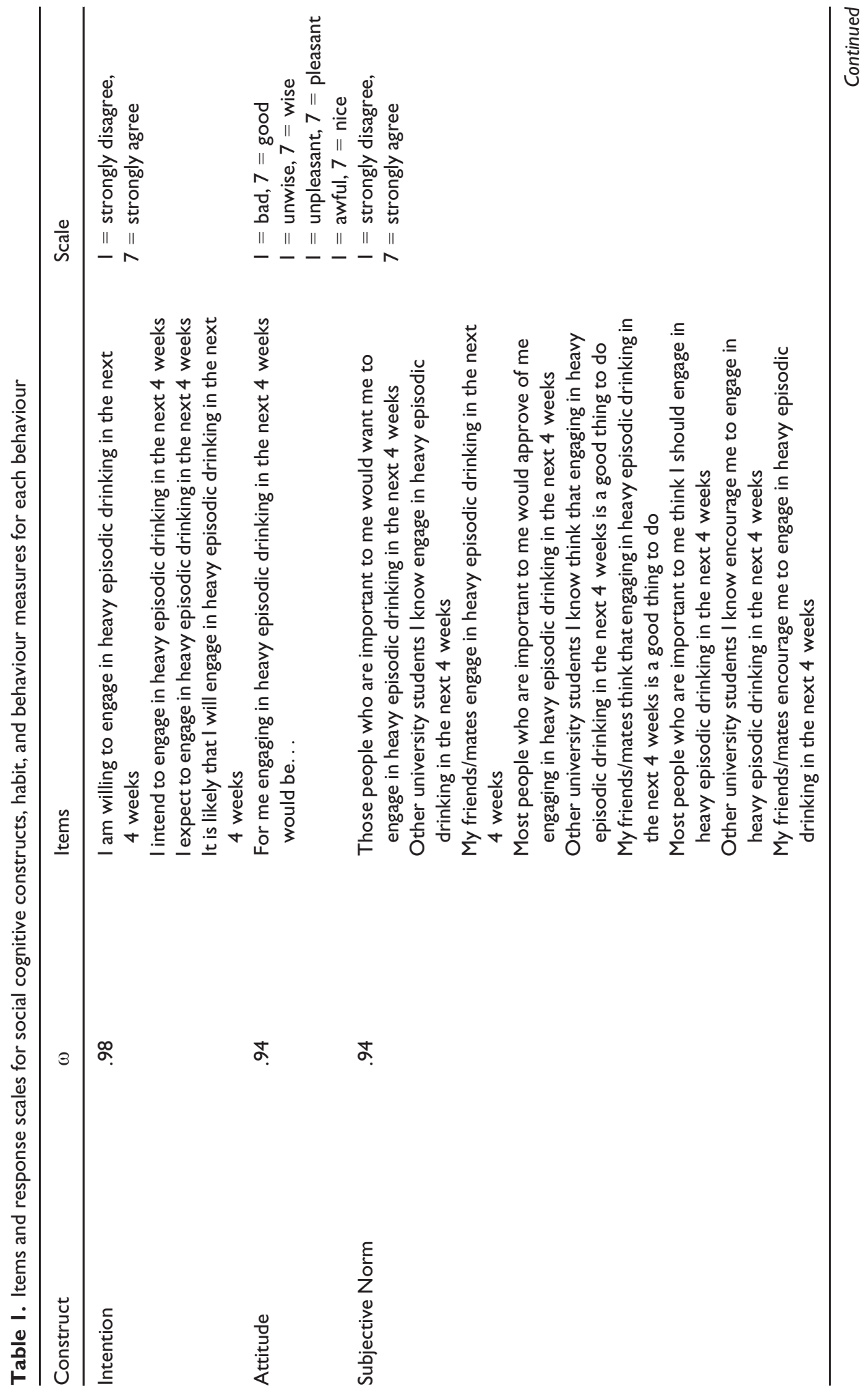




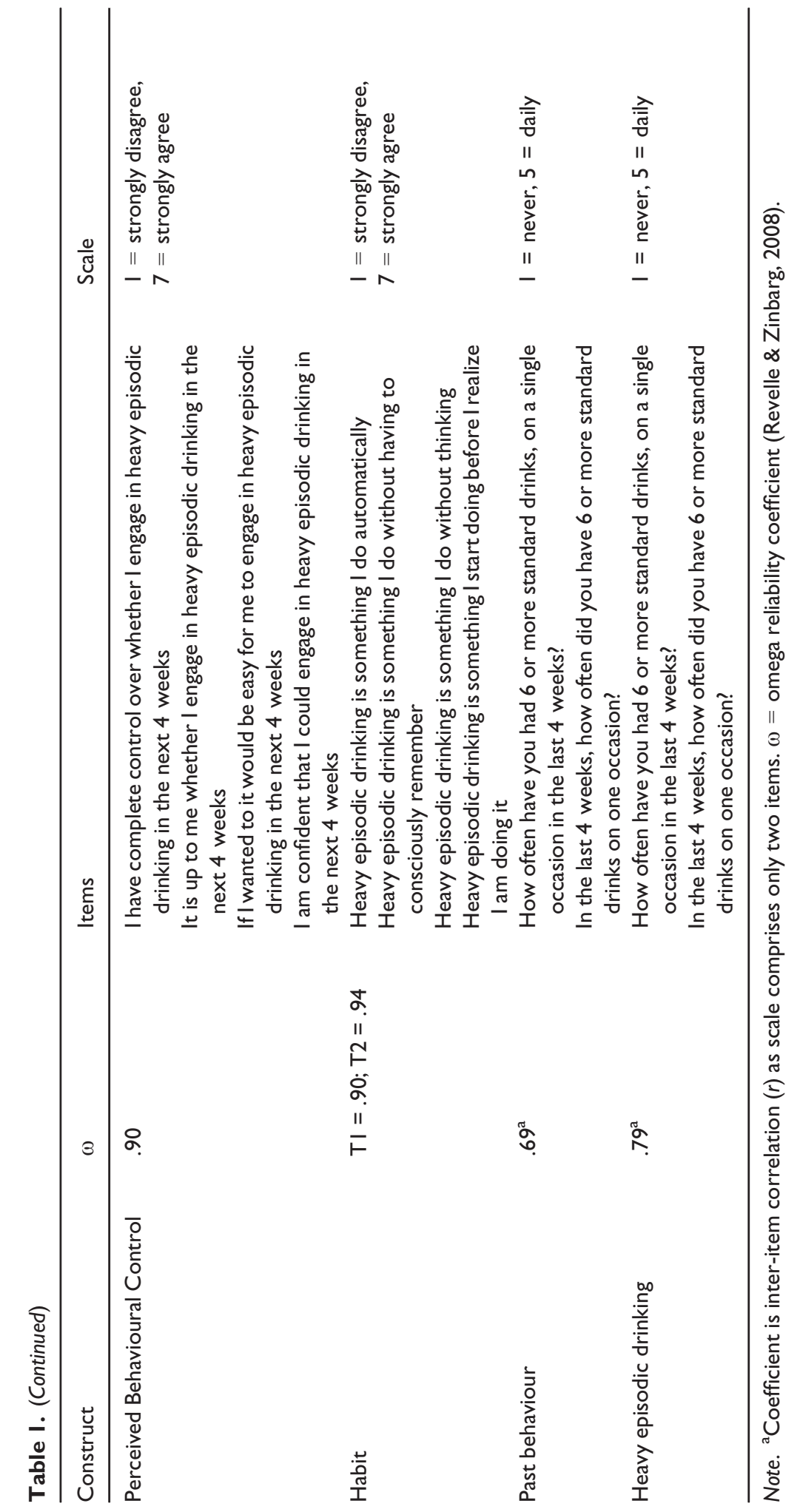


'For me to engage in heavy episodic drinking in the next 4 weeks would be: bad-good'), subjective norm (e.g., 'Those people who are important to me would want me to engage in heavy episodic drinking in the next 4 weeks'), and perceived behavioural control (e.g., 'I have complete control over whether I engage in heavy episodic drinking in the next 4 weeks').

Habit

Self-reported habit was measured using the 4-item self-reported behavioural automaticity index (e.g., 'Heavy episodic drinking is something I do automatically') (Gardner et al., 2012).

\section{Past behaviour}

Participants completed a two-item measure of their engagement in the target behaviour in the past month (e.g., 'How often have you had 6 or more standard drinks, on a single occasion in the last 4 weeks?').

\section{Demographic variables}

Participants self-reported their sex, age in years, and annual household income stratified by seven income levels based on national taxation brackets. A binary income variable was computed for use in analyses with $\geq \mathrm{AU} \$ 37,001$ as the cut-off for lower and middle-tohigh household income groups.

\section{Implicit alcohol identity}

Implicit alcohol identity was measured using the drinking identity implicit association test (DI-IAT) (Lindgren et al., 2015). Participants sort word stimuli into one of two categories (see Table 2), with response latencies used to calculate a $D$ score for each participant. Positive $D$ scores indicate words related to drinking being more quickly associated with words related to themselves in the sorting process, whereas negative $D$ scores are indicative of drinking words being more quickly associated with words not related to themselves (see Table 3 for details). $D$ scores were calculated using the improved scoring algorithm for the IAT (Greenwald, Nosek, \& Banaji, 2003; Lane, Banaji, Nosek, \& Greenwald, 2007). Specifically, the $D_{1}$ variant of the $D$ score was used. The scoring procedure involved trials with latency greater than $10,000 \mathrm{~ms}$ was deleted, and subjects where greater than $10 \%$ of trials were less than 300 ms were excluded. The inclusive $S D$ s between blocks 3 and 6 , and 4 and 7 were computed. Mean latencies for block 3, 4, 5, and 6 were computed. The mean latency for block 3 was subtracted from the mean latency for block 6 , and block 4 was subtracted from block 7 . The resultant difference scores were then divided by their associated inclusive standard deviation mentioned above, and these ratios averaged to compute the final $D$ score. Participants were required to correct errors before proceeding, so the optional error penalty was not applied (Lane et al., 2007).

\section{Data analysis}

Manifest measures of study constructs were computed by averaging item scores for each participant for each measure. Our hypothesized models were tested using path 


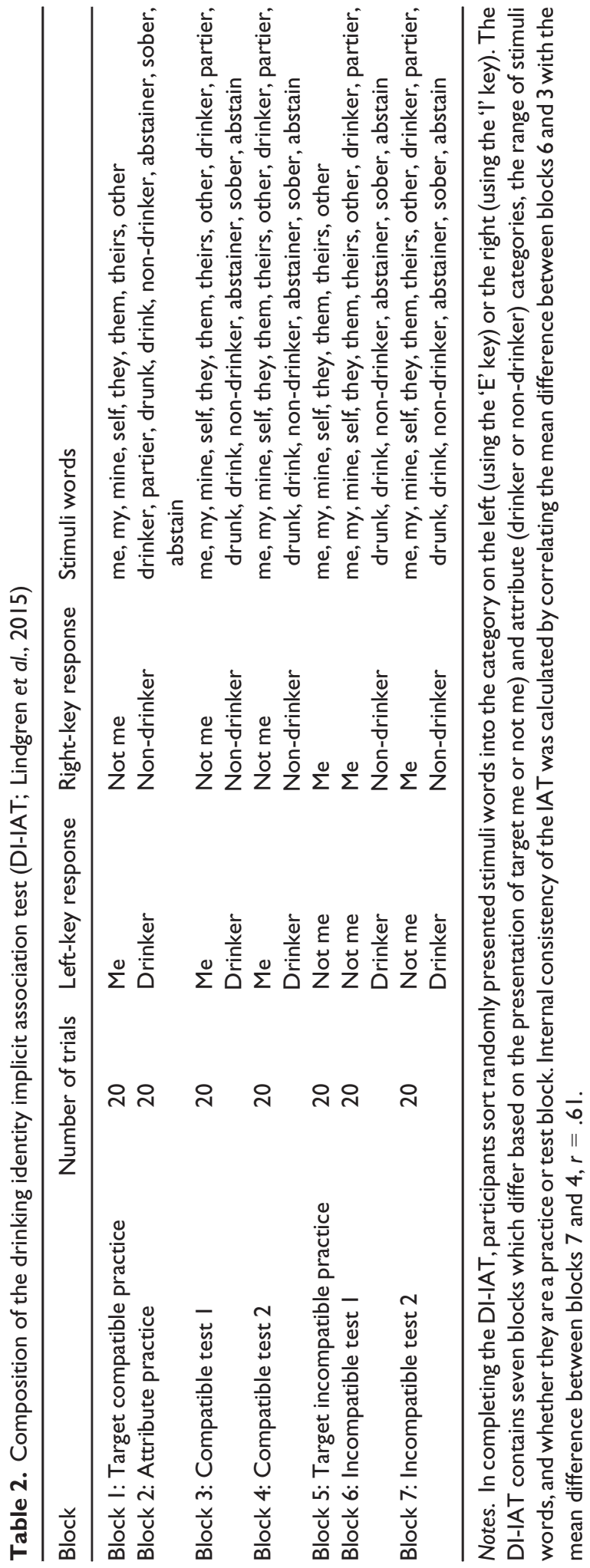




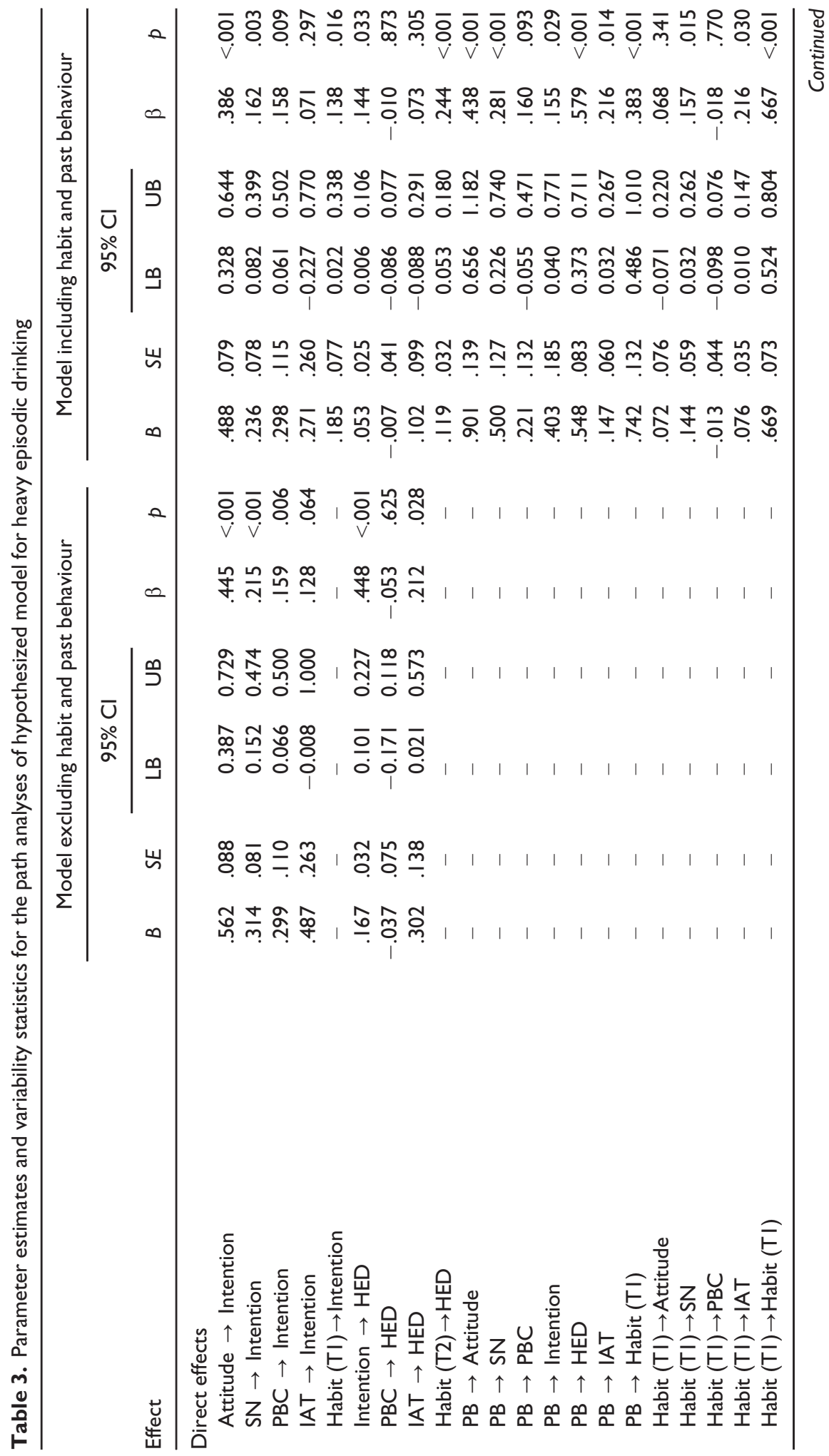




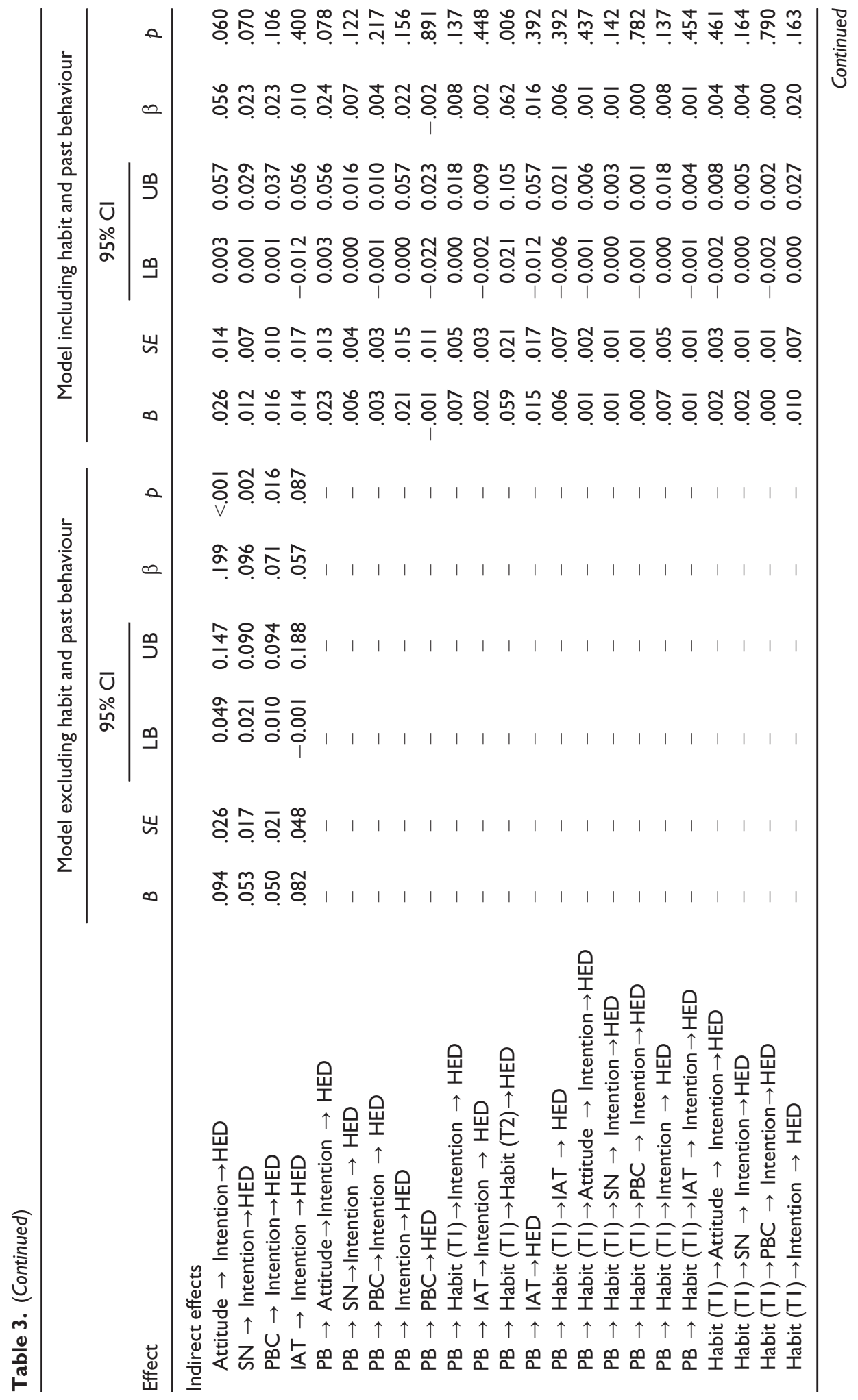




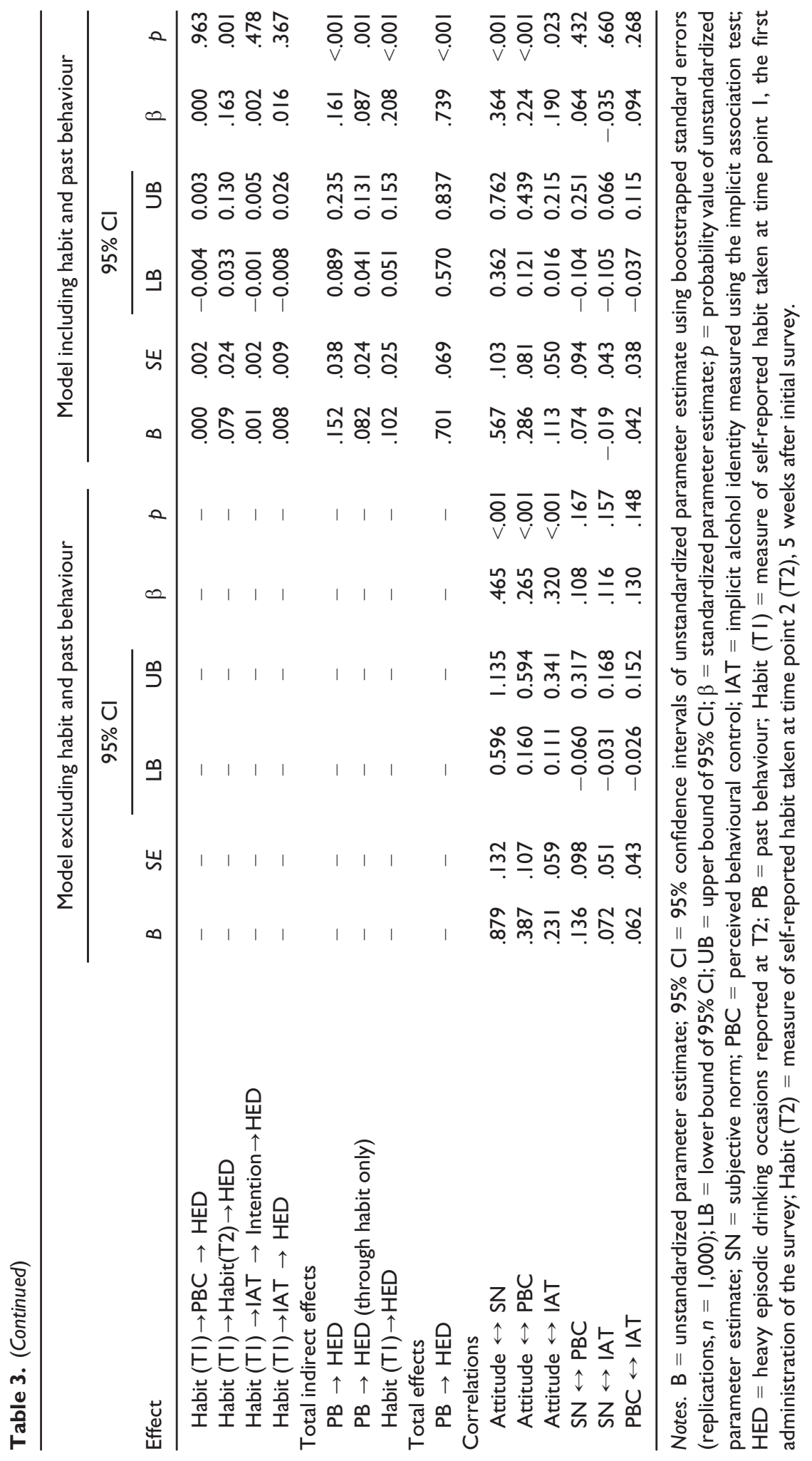


analysis with bootstrapped standard errors (Hayes, 2018). We specified two models. An initial model (Model 1) in which reasoned processes were represented by direct effects of the social cognitive constructs on intentions, intentions on follow-up HED behaviour, and indirect effects of the social cognitive constructs on behaviour through intentions. In addition, implicit alcohol identity was set as a direct predictor of behaviour, and an indirect predictor through intentions. These effects are summarized in Figure 1. We also estimated an augmented model (Model 2), which tested the effects of self-reported habit and past behaviour on relations among constructs in Model 1. The initial model was augmented to include direct effects of habit at the initial time point and at follow-up, and past frequency of HED, on follow-up behaviour. Indirect effects of past behaviour and habit on behaviour through the social cognitive constructs, intentions, and implicit alcohol identity were also estimated. The effects are presented in Figure 2. All model effects were controlled for sex, age, and income. We computed specific and total indirect effects using a maximum-likelihood estimation method with 1,000 bootstrap replications. Goodness of fit of the models with the data was evaluated using multiple criteria comparing the proposed model with the baseline model including the goodness-of-fit chi-square $\left(\chi^{2}\right)$ which should ideally be non-significant, the comparative fit index (CFI) which should exceed .95, the standardized root mean square residual (SRMR) which should be less than or equal to .08 , and the root mean square error of approximation (RMSEA) which should be below .05 with a narrow $90 \%$ confidence interval. Models were estimated using the lavaan package in $\mathrm{R}$ (Rosseel, 2012) with missing data imputed using the full information maximum-likelihood (FIML) method. The only missing data were the time 2 data for participants lost to follow-up. Little's test indicated that these data were missing completely at random. Simulation studies comparing approaches to handling missing data by Enders and Bandalos (Enders \& Bandalos, 2001) indicated FIML estimation is superior to deletion methods and response pattern imputation and that FIML provides unbiased estimates when data are missing at random or completely

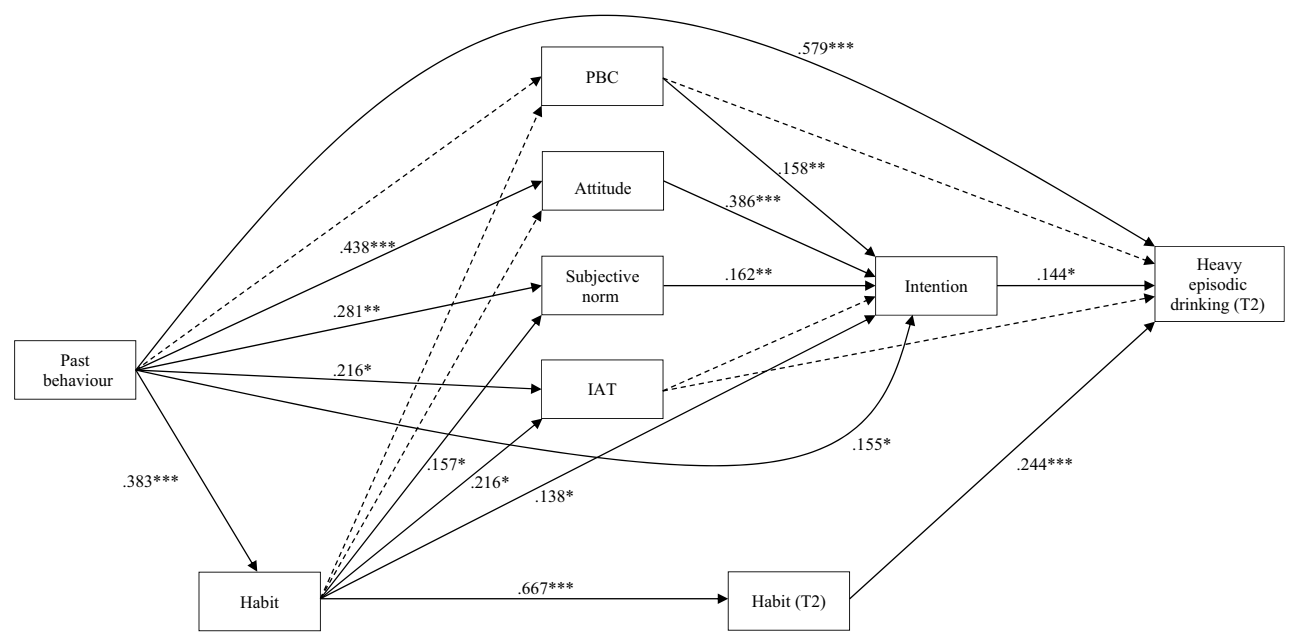

Figure 2. Standardized parameter estimates and probability statistics for path analysis of integrated dualprocess model including habit and past behaviour. T2 = Measure taken at time point 2, 4 weeks after the initial survey. ${ }^{* *} p<.00 \mathrm{I} ; * * p<.0 \mathrm{I} ; * p<.05$. 
at random. Data files and analysis scripts are available online from the Open Science Framework project for this study: https://osf.io/rj8ud.

\section{Results}

\section{Participants}

Of the participants who completed the initial survey $(N=204), 121$ (64.46\% female; mean age $=19.87$ years, $S D=2.11$ ) provided complete data for analysis after the second wave questionnaire ( $40.69 \%$ attrition rate). Tests for systematic differences in sample demographic characteristics due to attrition revealed no differences in sex distribution $\left(\chi^{2}=1.944, p=.163\right)$, income distribution $\left(\chi^{2}=2.391, p=.122\right)$, and age, $t$ $(202)=1.341, p=.181, d=.188$, between those who provided complete data at follow-up and those who dropped out or were eliminated due to a high proportion of missing data. Similarly, a MANOVA found no systematic differences due to attrition for the social cognitive (attitude, subjective norm, perceived behavioural control, intentions), self-reported habit, implicit alcohol identity, and self-reported HED variables, Wilks $\Lambda=0.976, F(7,143)=0.490, p=.841$. We also tested for differences in sample demographic characteristics and study variables across participants tested in the laboratory ( $n=42,34.71 \%)$ and those tested online $(n=79,65.29 \%)$ to provide a basis for pooling data from these groups of participants. We found no differences in sex $\left(\chi^{2}<.001, p=1.000\right)$ or income $\left(\chi^{2}=.029, p=.965\right)$ distribution between participants tested in the laboratory and those tested online. However, those tested in the laboratory $(M=20.69, S D=2.26)$ were slightly older than those tested in online $(M=19.43$, $S D=1.90), t(119)=3.250, p=.002, d=.591$. We also found no differences in the psychological and behavioural measures between participants tested in the laboratory and tested online, Wilks $\Lambda=0.924, F(7,113)=1.330, p=.242$.

\section{Model tests}

Statistically significant parameter estimates for Models 1 and 2 are presented in Figures 1 and 2, respectively. Full results of the path analytic models for each behaviour including unstandardized and standardized parameter estimates, confidence intervals, and test statistics for direct, indirect, and total effects are provided in Table 3. We also computed post-hoc statistical power using the WebPower package in R (Zhang \& Yuan, 2018) to ensure that we had sufficient power to detect desired effects. Power was estimated using Satorra and Saris' (1985) formula, with the sample size and degrees of freedom for each model, effect size estimated using the formula: $\chi^{2} /(n-1)$, and alpha set at 0.05 . Reproduced statistical power was .858 for Model 1 and .678 for Model $2{ }^{1}$

\section{Model I}

Model 1 tested the effects of social cognitive constructs from the theory of planned behaviour alongside our measure of implicit alcohol identity on HED (Figure 1). Although two of the incremental fit indices (CFI, SRMR) indicated good fit of the model with these data, the goodness-of-fit chi-square was statistically significant and the RMSEA exceeded

\footnotetext{
' Analysis scripts and output for the power analysis are available online from the Open Science Framework (OSF) project for this study: https://osf.io/rj8ud.
} 
recommended cut-off values, indicating some misfit, $\chi^{2}(2)=11.197, \quad p=.004$; $\mathrm{CFI}=.966 ;$ SRMR $=.027$; RMSEA $=.150,90 \% \mathrm{CI}=0.073,0.241) .^{2}$ Consistent with hypotheses, we found statistically significant effects of attitude, subjective norm, and perceived behavioural control on intentions, and intentions on HED. We also found significant indirect effects of attitude, subjective norm, and perceived behavioural control on HED mediated by intentions. Contrary to our predictions, however, perceived behavioural control did not predict behaviour directly. Consistent with our predictions, implicit alcohol identity was a statistically significant predictor of HED, with a small-tomedium sized effect, alongside the comparatively larger effect of intentions (Ajzen, 2011). There was no effect of implicit alcohol identity on intentions. Overall, the model accounted for significant proportions of the variance in intentions $\left(R^{2}=.497\right)$ and HED $\left(R^{2}=.299\right)$.

\section{Model 2}

In Model 2, introduced effects of self-reported habit at both time points and past behaviour are predictors of model constructs (Figure 2). The model fit the data well according to the multiple criteria adopted, $\chi^{2}(9)=12.459, p=.189 ;$ CFI $=.994$; SRMR $=.023$, RMSEA $=.043,90 \% \mathrm{CI}=0.000,0.096 .{ }^{3}$ We found statistically significant direct effects of past behaviour on attitude, subjective norm, implicit alcohol identity, intentions, habit at time 1, and HED. We also observed significant direct effects of habit at time 1 on implicit alcohol identity, intentions, subjective norms, and habit at time 2 . In addition, we found a significant direct effect of habit at time 2 on HED. Analysis of indirect effects revealed that effects of habit at time 1 on HED were directed through habit at time 2 , as predicted. Similarly, there was a significant indirect effect of past behaviour on HED through habit at both time points. We also found significant indirect effects of past behaviour through both habit and the social cognitive variables resulting in a significant total indirect effect of past behaviour on HED. The mediation proportion statistic $\left(\mathrm{P}_{\mathrm{M}}\right)$ (Ditlevsen, Christensen, Lynch, Damsgaard, \& Keiding, 2005) indicated that the indirect effect of past behaviour on HED through habit only accounted for a modest proportion of the total effect of past behaviour on HED $\left(\mathrm{P}_{\mathrm{M}}=.117\right)$, suggesting that the substantive proportion of the effect of past behaviour on HED was unaccounted for by habit. The social cognition constructs also accounted for a non-trivial, but relatively modest, proportion of the effect of past behaviour on HED $\left(\mathrm{P}_{\mathrm{M}}=.083\right)$. This indirect effect likely represents the extent to which past behaviour reflects previous reasoned, deliberation over

\footnotetext{
${ }^{2}$ Evaluation of the modification indices for this model indicated that model fit would be improved by adding direct effects of the attitude and subjective norm constructs on HED. Although these effects may have been tenable from a statistical perspective, they are not consistent with theory. Nevertheless, for completion we re-estimated the model to include these direct effects in an alternative model. Model fit was perfect as it was a saturated model. The model revealed a statistically significant effect of attitude on HED with a medium effect size, but a small non-significant effect of subjective norm on HED. The overall pattern of effects for intention and implicit alcohol identity remained, although the effects for both were smaller, and the effect for implicit alcohol identity fell marginally short of conventional alpha level for statistical significance $(p=.073)$. Results of this supplementary analysis can be found on (OSF) project for this study: https://osfio/rj8ud.

${ }^{3}$ As with Model I, we estimated an alternative model in which attitude and subjective norm were direct predictors of HED, along with habit at time I. Results revealed a well-fitting model, $\chi^{2}(2)=8.056, p=.234 ; C F I=.996 ; S R M R=.020$; RMSEA $=.041,90 \% \mathrm{Cl}=0.000,0.106$ ), with a small effect for attitude on HED which fell marginally short of the conventional alpha level for statistical significance $(p=.078)$, and a small non-significant effect for subjective norm. The overall pattern of effects was unchanged from the original model, with habit and past behaviour emerging as the only statistically significant predictors of HED. Results of this supplementary analysis can be found on (OSF) project for this study: https://osf.io/rj8ud.
} 
performing HED in the future. Finally, consistent with previous research applying social cognitive theories to predict health behaviour (Brown et al., 2017), including alcohol consumption (Caudwell et al., 2019; Norman \& Conner, 2006), introducing past behaviour and habit as predictors in the model attenuated model effects. Specifically, direct effects of intentions on HED, and indirect effects of attitude and subjective norm on HED through intentions, were significantly reduced with the inclusion of past behaviour and habit. In addition, the effect of implicit alcohol identity on HED was reduced in size so that it was no longer statistically significant. Tests of difference using Schenker and Gentleman's (Schenker \& Gentleman, 2001) standard method based on confidence intervals are available online from the Open Science Framework project for this study: https://osf.io/rj8ud.

\section{Discussion}

We tested an integrated model based on social cognitive and dual-processing theories to predict social drinkers' engagement in HED. Reasoned processes were represented by effects of the social cognition constructs and intentions from the theory of planned behaviour, and implicit processes were represented by effects of implicit alcohol identity measured using an implicit association test. We also included measures of self-reported habit and past behaviour, which can encompass information relating to both reasoned and implicit processes. An initial model indicated that drinkers' attitude and subjective norm predicted intentions, and intentions and implicit alcohol identity predicted participation in HED. These findings support the proposal that HED is a function of sets of beliefs that reflect reasoned and implicit processing. Inclusion of past behaviour and self-reported habit in the model revealed direct effects for habit and past behaviour on HED. Effects of time 1 habit on HED were indirect through time 2 habit, and there were also indirect effects of past behaviour on HED through habit at both time points and the social cognitive variables. The direct effects of intentions and implicit alcohol identity, and the indirect effects of attitude and subjective norm, on HED, were attenuated by the inclusion of habit and past behaviour.

Several notable implications for the determinants of HED emerged from this study, which extend previous research and may provide formative evidence on which to base behavioural interventions. First, the finding that implicit alcohol identity predicted behaviour unmediated by intentions is consistent with dual-process theories (Strack \& Deutsch, 2004) and previous research on alcohol, which suggests that implicit attitudes model non-conscious processes that determine behaviour (Caudwell et al., 2019). These implicit beliefs are likely to have been built up over time through regular positive experiences with the behaviour (e.g., enjoying drinking alcohol at someone's home, or in a club, or at a bar) which are likely activated or made highly accessible on presentation of relevant contexts (e.g., being in a bar with friends) (Hagger, 2020). This increases the likelihood that individuals holding such implicit beliefs will participate in drinking behaviour with very little cognitive input required when they find themselves in that context. HED for university students is likely a highly rewarding behaviour regulated by previously learned behavioural patterns. However, the fact that intentions also predicted behaviour suggests that HED is also determined by reasoned decision-making. A potential interpretation of these data is that some individuals are more likely to engage in HED as a consequence of an implicit process, while others' decisions are determined by reasoned processes. 
Attenuation of the effects of the constructs representing reasoned processes that lead to behaviour such as intentions, attitude, and subjective norm by past behaviour and habit suggests that risky alcohol consumption is strongly influenced by previous experience and habit. The attenuating effects of past behaviour and habit on effects of these constructs have been consistently observed in previous research applying social cognitive theories (for a meta-analyses, see Hagger, Chan, Protogerou, \& Chatzisarantis, 2016; Hagger, Polet, \& Lintunen, 2018). Findings indicate that observed effects of social cognition variables on behaviour in previous research are likely inflated in the absence of habits and past behaviour, and may give a misleading assessment on the extent to which behaviours, such as HED, are predicted by constructs representing reasoned processes. In addition, the effect of implicit alcohol identity was also attenuated to a smaller value indicating that habits and past behaviour may largely account for the effects of implicit beliefs. These findings indicated that implicit alcohol identity, like many implicit measures, is likely to reflect representations of the behaviour developed through experience. As before, implicit alcohol identity is likely developed over time through consistent experiences of alcohol consumption with similar accompanying self-related evaluations (Abelson, 1981; Hagger, 2019). As a consequence, although implicit identity is a direct determinant of HED, current data indicate that this effect is largely dependent on past experience, an unsurprising finding given that frequency of past behaviour is an important, although not the only, component of habit (Ouellette \& Wood, 1998; Wood \& Rünger, 2016). These findings, at least, shed light on the potential reason why implicit alcohol identity accounts for variance in HED. Nevertheless, it would be important to establish whether such implicit measures predict behaviour in the face of past behaviour in novice heavy episodic drinkers, and among those with different, more moderate drinking patterns.

The indirect effect of past behaviour through habit on HED suggests past risky drinking behaviour is, at least in part, a function of habits. Previous research has suggested that past behaviour serves to summarize the effects of habits on behaviour. For example, studies have demonstrated that past behaviour effects are much larger for behaviours that individuals perform regularly in the presence of stable contexts or cues, such as drinking heavily when out with friends at a bar (Ouellette \& Wood, 1998). Habits, therefore, would be expected to mediate effects of past behaviour on subsequent behaviour, an observation found in the current study and in previous research (van Bree et al., 2015). However, it should be noted that only a small proportion of the effect of past behaviour on HED is accounted for by habit. It could be that the residual effect of past behaviour on HED may reflect unmeasured constructs that affect behaviour independent of intentions and other social cognition constructs. Examples of these constructs might be implicitly held beliefs or individual differences that predispose individuals' impulsive behavioural patterns such as trait self-control, impulsivity, and certain personality traits (Allom et al., 2018; Hagger, Gucciardi, Turrell, \& Hamilton, 2019).

The present research has numerous strengths including a focus on HED, a common yet risky drinking behaviour among young university students, and the adoption of an integrated dual-process theoretical approach and appropriate measures. However, several limitations should be acknowledged. HED behaviour was measured using a relatively brief self-report measure which may be subject to recall and social desirability bias. The use of more comprehensive methods such as the timeline follow-back technique, ecological momentary assessment, or breathalysing individuals may provide more accurate estimates. One of our measures reflecting implicit determinants of HED was implicit alcohol identity. We selected this measure over, for example, implicit 
attitudes towards alcohol, because we considered it more closely linked to the act of drinking. However, this contrasts with previous research which has focused on implicit attitudes towards the substance in question (e.g., implicit attitudes towards sugar; Hagger, Trost, Keech, Chan, \& Hamilton, 2017). Future research should consider testing the relative effects of IATs tapping implicit attitudes towards alcohol and implicit identities on alcohol consumption. It is also important to note that although effects of past behaviour and habit are considered as reflecting non-conscious implicit effects, the fact that we found indirect effects of these constructs on HED indicates that they may model both reasoned and implicit effects. In addition, the assumption that the residual effects of these variables on HED reflect non-conscious processes should also be interpreted with caution as the intention and other social cognition constructs in the current study may not have sufficiently captured all aspects of habit and past behaviour attributable to reasoned processed. Further, current findings are based on theory alone as the study design did not permit inferences of directionality or causation. The use of longitudinal designs measuring all variables across several time points and the estimation of a cross-lagged paned model would allow the direction of effects to be empirically determined in future tests. While preliminary analyses revealed no significant differences in demographic or baseline psychological variables between those who completed the follow-up and those who did not, it should be noted that the relatively high attrition rate in the current study is a potential limitation. It is also important to note that constructs that represented reasoned and implicit processes were confined to those based on theory (Ajzen, 2011; Strack \& Deutsch, 2004) and prior research (Caudwell et al., 2019; Hagger et al., 2017), which may not fully account for the non-conscious processes that relate to HED. In addition, measures such as the self-report habit index are meta-cognitive measures, which reflect implicit, non-conscious processes rather than tap them directly (cf., Hagger, Rebar, Mullan, Lipp, \& Chatzisarantis, 2015; Sniehotta \& Presseau, 2012). Investigation of other constructs that may account for, or represent, non-conscious processes in HED such as trait self-control (Hagger, Hankonen, et al., 2019), emotional processes (Conner, McEachan, Taylor, O'Hara, \& Lawton, 2015), and individual difference and personality factors (Cyders, Flory, Rainer, \& Smith, 2009; Vo \& Bogg, 2015) should be considered in future research. Finally, statistical power for our final model including past behaviour and habit fell marginally short of typically recommended criterion for adequate power to detect effects. This highlights the imperative for future replications of the current model in larger samples.

\section{Conclusion}

Drawing from theories of social cognition and dual-process models, the present study tested key hypotheses for effects of constructs that represent reasoned and implicit pathways to HED in a sample of Australian university students who drink alcohol. Current results indicate that university students' HED is predicted by past behaviour and habit. Direct effects of habit and past behaviour unmediated by intentions have often been interpreted as representing effects of non-conscious processes on behaviour (e.g., Hagger et al., 2018; Ouellette \& Wood, 1998). However, it is important to note that measures of intentions and social cognition constructs may not be sufficient in capturing all the information encompassed by habit and past behaviour attributable to reasoned processes. Furthermore, direct effects of these variables on behaviour may also represent effects of unmeasured constructs that reflect reasoned processes. So, solely attributing effects of habit and past behaviour on HED to non-conscious processes should be interpreted with 
caution. Current findings should be viewed as preliminary and require further replication. However, they may signpost possible avenues for the development of behaviour change interventions. For example, interventionists seeking to reduce rates of HED in students may consider strategies that assist in minimizing exposure to contexts or cues that activate implicit alcohol identities such as minimizing availability and creating social events that do not revolve around alcohol consumption, and strategies to provide students with selfregulatory skills such as planning alternative courses of action when in tempting situations (e.g., Duckworth, Gendler, \& Gross, 2016; Hagger, 2019; Hollands, Marteau, \& Fletcher, 2016). Further research is needed to identify factors that further account for the pervasive effects of past behaviour on HED.

\section{Acknowledgements}

Martin S. Hagger's contribution was supported by a Finland Distinguished Professor (FiDiPro) award (Dnro 1801/31/2105) from Business Finland. We would also like to thank Andrew Campbell for his assistance with data matching and preparation.

\section{Conflicts of interest}

The authors declare that they have no conflict of interest.

\section{Funding}

This research did not receive any specific grant from funding agencies in the public, commercial, or not-for-profit sectors.

\section{Data availability statement}

Data files and analysis scripts are available online from the Open Science Framework project for this study: https://osf.io/rj8ud.

\section{References}

Abelson, R. P. (1981). Psychological status of the script concept. American Psychologist, 36, 715-729. https://doi.org/10.1037/0003-066X.36.7.715

Ajzen, I. (2002a). Constructing a TPB questionnaire: Conceptual and methodological considerations. (September 1, 2002). Retrieved from http://people.umass.edu/ aizen/pdf/ tpb.measurement.pdf

Ajzen, I. (2002b). Residual effects of past on later behavior: Habituation and reasoned action perspectives. Personality and Social Psychology Review, 6, 107-122. https://doi.org/10.1207/ S15327957PSPR0602_02

Ajzen, I. (2011). The theory of planned behaviour: Reactions and reflections. Psychology and Health, 26, 1113-1127. https://doi.org/10.1080/08870446.2011.613995

Allom, V., Mullan, B., Cowie, E., \& Hamilton, K. (2016). Physical activity and transitioning to college: The importance of intentions and habits. American Journal of Health Behavior, 40, 280-290. https://doi.org/10.5993/AJHB.40.2.13

Allom, V., Mullan, B. A., Monds, L., Orbell, S., Hamilton, K., Rebar, A., \& Hagger, M. S. (2018). Reflective and impulsive processes underlying saving behaviour and the additional roles of selfcontrol and habit. Journal of Neuroscience, Psychology, and Economics, 11, 135-146. https:// doi.org/10.1037/npe0000093 
Brown, D. J., Hagger, M. S., Morrissey, S., \& Hamilton, K. (2017). Predicting fruit and vegetable consumption in long-haul heavy goods vehicle drivers: Application of a multi-theory, dual-phase model and the contribution of past behaviour. Appetite, 121,326-336. https://doi.org/10.1016/ j.appet.2017.11.106

Caudwell, K. M., Keech, J. J., Hamilton, K., Mullan, B. A., \& Hagger, M. S. (2019). Reducing alcohol consumption during pre-drinking sessions: Testing an integrated behaviour-change model. Psychology \& Health, 34, 106-127. https://doi.org/10.1080/08870446.2018.1518527

Conner, M. T., McEachan, R., Taylor, N., O'Hara, J., \& Lawton, R. (2015). Role of affective attitudes and anticipated affective reactions in predicting health behaviors. Health Psychology, 34, 642652. https://doi.org/10.1037/hea0000143

Cyders, M. A., Flory, K., Rainer, S., \& Smith, G. T. (2009). The role of personality dispositions to risky behavior in predicting first-year college drinking. Addiction, 104, 193-202. https://doi.org/10. 1111/j.1360-0443.2008.02434.x

Ditlevsen, S., Christensen, U., Lynch, J., Damsgaard, M. T., \& Keiding, N. (2005). The mediation proportion: A structural equation approach for estimating the proportion of exposure effect on outcome explained by an intermediate variable. Epidemiology, 16, 114-120. https://doi.org/10. 1097/01.ede.0000147107.76079.07

Duckworth, A. L., Gendler, T. S., \& Gross, J. J. (2016). Situational strategies for self-control. Perspectives on Psychological Science, 11, 35-55. https://doi.org/10.1177/17456916156 23247

Enders, C. K., \& Bandalos, D. L. (2001). The relative performance of full information maximum likelihood estimation for missing data in structural equation models. Structural Equation Modeling, 8, 430-457. https://doi.org/10.1207/S15328007SEM0803_5

Gardner, B., Abraham, C., Lally, P., \& de Bruijn, G.-J. (2012). Towards parsimony in habit measurement: Testing the convergent and predictive validity of an automaticity subscale of the self-report habit index. International Journal of Behavioral Nutrition and Physical Activity, 9, 102. https://doi.org/10.1186/1479-5868-9-102

Greenwald, A. G., Nosek, B. A., \& Banaji, M. R. (2003). Understanding and using the Implicit Association Test: I. An improved scoring algorithm. Journal of Personality and Social Psychology, 85, 197-216. https://doi.org/10.1037/0022-3514.85.2.197

Hagger, M. S. (2019). Habit and physical activity: Theoretical advances, practical implications, and agenda for future research. Psychology of Sport and Exercise, 42, 118-129. https://doi.org/10. 1016/j.psychsport.2018.12.007

Hagger, M. S. (2020). Redefining habits and linking habits with other implicit processes. Psychology of Sport and Exercise, 46, 101606. https://doi.org/10.1016/j.psychsport.2019.101606

Hagger, M. S., Chan, D. K. C., Protogerou, C., \& Chatzisarantis, N. L. D. (2016). Using meta-analytic path analysis to test theoretical predictions in health behavior: An illustration based on metaanalyses of the theory of planned behavior. Preventive Medicine, 89, 154-161. https://doi.org/ 10.1016/j.ypmed.2016.05.020

Hagger, M. S., Gucciardi, D. F., Turrell, A., \& Hamilton, K. (2019). Self-control and health-related behavior: The role of implicit self-control, trait self-control, and lay beliefs in self-control. British Journal of Health Psychology, 24, 764-786. https://doi.org/10.1111/bjhp.12378

Hagger, M. S., Hankonen, N., Kangro, E.-M., Lintunen, T., Pagaduan, J., Polet, J., . . Hamilton, K. (2019). Trait self-control, social cognition constructs, and intentions: Correlational evidence for mediation and moderation effects in diverse health behaviors. Applied Psychology: Health and Well-Being, 11, 407-437. https://doi.org/10.1111/aphw.12153

Hagger, M. S., Polet, J., \& Lintunen, T. (2018). The reasoned action approach applied to health behavior: Role of past behavior and test of some key moderators using meta-analytic structural equation modeling. Social Science and Medicine, 213, 85-94. https://doi.org/10.1016/j.socsc imed.2018.07.038

Hagger, M. S., Rebar, A. L., Mullan, B. A., Lipp, O. V., \& Chatzisarantis, N. L. D. (2015). The subjective experience of habit captured by self-report indexes may lead to inaccuracies in the measurement 
of habitual action. Health Psychology Review, 9, 296-302. https://doi.org/10.1080/17437199. 2014.959728

Hagger, M. S., Trost, N., Keech, J., Chan, D. K. C., \& Hamilton, K. (2017). Predicting sugar consumption: Application of an integrated dual-process, dual-phase model. Appetite, 116, 147156. https://doi.org/10.1016/j.appet.2017.04.032

Hamilton, K., Kirkpatrick, A., Rebar, A., \& Hagger, M. S. (2017). Child sun safety: Application of an integrated behavior change model. Health Psychology, 36, 916-926. https://doi.org/10.1037/ hea0000533.

Hayes, A. F. (2018). Introduction to mediation, moderation, and conditional process analysis: A regression-based approach (2nd ed.). New York, NY: Guildford Press.

Hollands, G. J., Marteau, T. M., \& Fletcher, P. C. (2016). Non-conscious processes in changing healthrelated behaviour: A conceptual analysis and framework. Health Psychology Review, 10, 381394. https://doi.org/10.1080/17437199.2015.1138093

Lane, K. A., Banaji, M. R., Nosek, B. A., \& Greenwald, A. G. (2007). Understanding and using the Implicit Association Test: IV. What we know (so far). In B. Wittenbrink, \& N. S. Schwarz (Eds.), Implicit measures of attitudes: Procedures and controversies (pp. 59-102). New York, NY: Guildford Press.

Lindgren, K. P., Neighbors, C., Teachman, B. A., Gasser, M. L., Kaysen, D., Norris, J., \& Wiers, R. W. (2015). Habit doesn't make the predictions stronger: Implicit alcohol associations and habitualness predict drinking uniquely. Addictive Behaviors, 45, 139-145. https://doi.org/ 10.1016/j.addbeh.2015.01.003

NHMRC. (2009). Australian Guidelines to reduce bealth risks from drinking alcohol. Canberra, ACT: Author.

Norman, P., \& Conner, M. (2006). The theory of planned behaviour and binge drinking: Assessing the moderating role of past behaviour within the theory of planned behaviour. British Journal of Health Psychology, 11, 55-70. https://doi.org/10.1348/135910705X43741

Orbell, S., \& Verplanken, B. (2010). The automatic component of habit in health behavior: Habit as cue-contingent automaticity. Health Psychology, 29, 374-383. https://doi.org/10.1037/ a0019596

Ouellette, J. A., \& Wood, W. (1998). Habit and intention in everyday life: The multiple processes by which past behavior predicts future behavior. Psychological Bulletin, 124, 54-74. https://doi. org/10.1037//0033-2909.124.1.54

Prosser, T., Gee, K. A., \& Jones, F. (2018). A meta-analysis of effectiveness of E-interventions to reduce alcohol consumption in college and university students. Journal of American College Health, 66, 292-301. https://doi.org/10.1080/07448481.2018.1440579

Revelle, W., \& Zinbarg, R. E. (2008). Coefficients Alpha, Beta, Omega, and the glb: Comments on Sijtsma. Psychometrika, 74, 145. https://doi.org/10.1007/s11336-008-9102-z

Rosseel, Y. (2012). lavaan: An R package for structural equation modeling. Journal of Statistical Software, 48, 1-36. https://doi.org/10.18637/jss.v048.i02

Satorra, A., \& Saris, W. E. (1985). Power of the likelihood ratio test in covariance structure analysis. Psychometrika, 50, 83-90. https://doi.org/10.1007/bf02294150

Schenker, N., \& Gentleman, J. F. (2001). On judging the significance of differences by examining the overlap between confidence intervals. The American Statistician, 55, 182-186. https://doi.org/ $10.1198 / 000313001317097960$

Sniehotta, F. F., \& Presseau, J. (2012). The habitual use of the self-report habit index. Annals of Behavioral Medicine, 43, 139-140. https://doi.org/10.1007/s12160-011-9305-x

Stafford, J. K. D. (2017). Alcohol in the university setting: A resource to support Australian universities. Perth, WA: McCusker Centre for Action on Alcohol and Youth, Curtin University.

Strack, F., \& Deutsch, R. (2004). Reflective and impulsive determinants of social behavior. Personality and Social Psychology Review, 8, 220-247. https://doi.org/10.1207/ s15327957pspr0803_1

van Bree, R. J. H., van Stralen, M. M., Mudde, A. N., Bolman, C., de Vries, H., \& Lechner, L. (2015). Habit as mediator of the relationship between prior and later physical activity: A longitudinal 
study in older adults. Psychology of Sport and Exercise, 19, 95-102. https://doi.org/10.1016/j. psychsport.2015.03.006

Vo, P. T., \& Bogg, T. (2015). Testing theory of planned behavior and neo-socioanalytic theory models of trait activity, industriousness, exercise social cognitions, exercise intentions, and physical activity in a representative U.S. sample. Frontiers in Psychology, 6, 1114. https://doi.org/10. $3389 /$ fpsyg.2015.01114

WHO. (2018). Global status report on alcohol and health 2018. Geneva, Switzerland: World health Organization.

Wood, W., \& Rünger, D. (2016). Psychology of habit. In S. T. Fiske (Ed.), Annual review of psychology (Vol. 67, pp. 289-314). Palo Alto, CA: Annual Reviews.

Zhang, Z., \& K.-H. Yuan (Eds.) (2018). Practical statistical power analysis using Webpower and $R$. Granger, IN: ISDSA Press.

Received 24 April 2019; revised version received I8 November 2019 